\title{
Mini Review - Understanding Chemotherapy Induced Neuropathy from Two Points of View
}

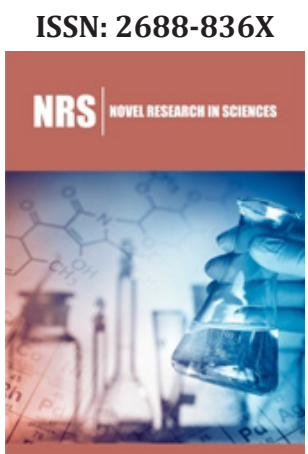

*Corresponding author: Ali Alshahrani, Associate Professor, Department of Clinical Pharmacy, Taif University Al Huwaya, Taif 26571, Saudi Arabia

Submission: 师眥 July 28, 2021

Published: 海August 13, 2021

Volume 9 - Issue 1

How to cite this article: Ali Alshahrani. Mini Review - Understanding Chemotherapy Induced Neuropathy from Two Points of View. Nov Res Sci. 9(1). NRS. 000702. 2021.

DOI: $10.31031 /$ NRS.2021.09.000702

Copyright@ Ali Alshahrani, This article is distributed under the terms of the Creative Commons Attribution 4.0 International License, which permits unrestricted use and redistribution provided that the original author and source are credited.

\author{
Ali Alshahrani* \\ Department of Clinical Pharmacy, Taif University Al Huwaya, Taif 26571, Saudi Arabia
}

\begin{abstract}
It is important to understand Chemotherapy induced neuropathy from two points of view. From the patient's point of view, and from the point of view of a provider. In both perspectives, it is important to articulate the common CIPN presentations, as well as describe their clinical significance, and recognize the diagnostic conundrums, which arise whenever new neuropathies arise in cancer patients. Such knowledge helps to directly CIPN guidelines into patient care. Noting that CIPN occurs in patients, going forward, it is more important to determine why we ought to care about chemotherapy induced neuropathy.
\end{abstract}

Keywords: Cryotherapy; Cooling caps; Neuropathy; Toxic; Nerves; Spinal cord; Chemotherapy

Abbreviation: CIPN: Chemotherapy Induced Peripheral Neuropathy

\section{Introduction}

Common talk about neuropathy pertains patients and providers. From the perspective of a patient, the most important elements concern how to recognize neuropathy symptoms, risk factors, and prevention strategies, the therapeutic options available, and how to recognize when the patient needs an expert. Neuropathy incidences increase over time especially with age. Neuropathy simply means damage sustained by nerves. Basically, when discussing neuropathy, the discussion revolves about nerves, which begin in the spinal cord, and descend down to the limbs. Sensory receptors on the skin and in all the tissues receive information from nerves. Motor nerves control muscles and how people use their strength [1].

\section{Discussion}

Most neuropathies are length dependent. Since the body is close to the spinal cord, anything affecting the nerves or anything that poison the nerve or damage it, makes it difficult for the nerves to send nutrients or signals to the nerves. So, when people develop neuropathy, it often begins from their fingertips and toes and can slowly work its way backwards. Most chemotherapy induced neuropathy takes this form or another way, which people lose sensation, trouble with dexterity, and trouble with balance, or experience a substantial amount of pain in the event it affects their motor neurons. CIPN mostly affects the autonomic nerves-and these are nerves responsible for supplying a lot of organs in the body. In short, CIPN is a toxic neuropathy, which means that it originates from the toxin, which is chemotherapy. In short, the more the chemotherapy administered, the worse the neuropathy possible. This is the underlying principle. Sometimes, another strange phenomenon happens, called coasting. Here, owing to some chemotherapeutic agents, patients may develop, or may not develop neuropathy during chemotherapy, however, even after chemotherapy stops, one month or three months down, a patient may still develop certain nerve symptoms previously absent. However, this occurs in a minority of patients. One of the most important questions today concerning chemotherapy is that chemotherapy is a cure for cancer. Knowing this, then why do we care so much about chemotherapy induced neuropathy?

\section{Conclusion}

Patients want to know answers to important questions related to neuropathy. These need to be the focus of studies because, since treatment to cure cancer causes neuropathy, 
patients want to know what next after the neuropathies. The first question of interest is where have we come in relation to cancer treatment in the past five years? There is some progress in cancer treatment, some of which, are medicine, some are not. For instance, cryotherapy without or with compression, patients wear cooling caps on their heads while receiving chemotherapy treatment for prevention of hair loss. This strategy serves two important functions. Cooling the skin reduces the amount of blood flow and reduces the amount of blood exposed to chemotherapy. Colling down also slows down metabolism on tissues reducing the amount of tissue injury, leading to significant reduction in sensation loss, and reduction of pain among patients [2].

Another important question comes from patients wanting to know whether they will recover. Although patients may develop neuropathy in the course of treatment, they recover quickly; some may plateau but will eventually recover from their neuropathies. Even for patients that experience coasting phenomenon, there is still room for eventual recovery from neuropathy. While it's difficult to determine the end point of neuropathies, it is assuring to tell patients that they will eventually recover. While people may take up to two years to recover from neuropathy, it is important to have them recover from it as fast as possible. This is of utmost importance and worth exploring moving forward.

\section{Acknowledgement}

The author thanks the college of pharmacy, Taif University for the scientific support.

\section{References}

1. Shin GJE, Pero ME, Hammond LA, Burgos A, Kumar A, et al. (2021) Integrins protect sensory neurons in models of paclitaxel-induced peripheral sensory neuropathy. Proceedings of the National Academy of Sciences 118(15).

2. Kate S, Patil R, Pathan D, Vyavhare R, Joseph S, et al. (2021) Safety and efficacy of scalp cooling system in preventing chemotherapy induced alopecia-A single center prospective study. Cancer Treatment and Research Communications 26: 100280. 